\title{
Comparison of the effects of temporomandibular joint and cervical vertebra treatment on pain and functional improvement in persons with tension-type headaches
}

\author{
Junghyun Kwon ${ }^{a}$, Wonjong Yu ${ }^{b}$ \\ ${ }^{a}$ Department of Physical Therapy, Graduate School, Eulji University, Seongnam, Republic of Korea \\ ${ }^{b}$ Department of Physical Therapy, Eulji University, Seongnam, Republic of Korea
}

Objective: The purpose of this study was to evaluate the effects of temporomandibular joint and cervical vertebra treatment in persons with tension-type headaches on pain, tenderness, and functional improvement.

Design: Three-group pretest-posttest design.

Methods: Subjects with tension-type headaches were divided into the temporomandibular joint and cervical vertebra treatment group $(n=11)$, temporomandibular joint treatment group $(n=11)$, and cervical vertebra treatment group ( $n=11)$, and pre- and post-evaluation was performed. The temporomandibular joint treatment group underwent compression massage and joint ply of the muscles around the temporomandibular joint. The cervical vertebra group received deep tendon massage and Myofascial Release of the cervical muscles. The temporomandibular joint and cervical vertebra treatment group performed both types of treatment. Treatment was performed for 50 minutes, three times a week for 4 weeks. Measurement tools included the Korean version of the short form-McGill Pain Questionnaire (SF-MPQ, K), Headache Impact test-6 (HIT-6), Neck Disability Index (NDI), and the Digital Algometer FPX25.

Results: The groups showed significant differences in SF-MPQ, HIT-6 test, NDI, and Alogometer FPX25 test scores before and after intervention $(p<0.05)$. The differences between the groups were most significant in the group that received treatment of the temporomandibular joint and cervical vertebra $(p<0.05)$.

Conclusions: In this study, the treatment of the temporomandibular joint and cervical vertebra was shown to be effective for improving pain, quality of life, and cervical vertebra in persons with tension-type headaches. This data may be helpful in identifying treatment techniques for tension-type headaches in the future.

Key Words: Cervical vertebra, Pain, Temporomandibular joint, Tension-type headache

\section{Introduction}

Headache is defined as pain in the head and cervical region and is one of the most commonly reported symptoms in the adult population [1,2]. Headache discomfort can have social costs on patients and increase the overall burden on society, beyond individual and family problems. Consequently, the World Health Organization (WHO) defines headaches as one of the top 10 threats to human health
(WHO, 2007). Headaches are classified into groups including migraine, tension-type headache, chronic paroxysmal migraine, various headaches without structural lesions, trauma, cerebrovascular disorder, or extracranial infection, etc., according to the classification of the International Headache Society (1988). A primary headache is one where the primary diagnosis is given for a headache, whereas headaches that occur along with other diseases are called secondary headaches [3].

Received: 25 November, 2019 Revised: 18 December, 2019 Accepted: 18 December, 2019

Corresponding author: Wonjong Yu (ORCID https://orcid.org/0000-0002-8119-1093)

Department of Physical Therapy, Eulji University, 553 Sanseong-daero, Sujeong-gu, Seongnam 13135, Republic of Korea

Tel: 82-31-740-7385 Fax: 82-31-740-7367 E-mail: wjyu@eulji.ac.kr

(c) This is an Open-Access article distributed under the terms of the Creative Commons Attribution Non-Commercial License (http://creativecommons.org/licenses/ by-nc/4.0) which permits unrestricted non-commercial use, distribution, and reproduction in any medium, provided the original work is properly cited.

Copyright @ 2019 Korean Academy of Physical Therapy Rehabilitation Science 
A tension-type headache, which is a primary headache, has no known cause, but it creates pain by causing muscle contraction of the head and neck area due to stress, tension, and overwork. This pain typically spreads from the back of the head and down caudally [4,5]. Although drug treatment is commonly used and is effective for treating tension-type headaches, there are a large number of patients in whom this treatment is ineffective. In addition, patients who rely on drugs for treatment are at risk of rebound headaches and tend to suffer from chronic headaches [6].

In a previous study, the effects of tension-type headaches on the cervical vertebra using joint mobility techniques were investigated [7]. In this study, high-frequency deep diathermy on the posterior head muscle was applied for tension-type headaches, impaired neck mobility and dysfunction [8]. In addition, the effects of cervical vertebra treatment on tension-type headache patients were investigated [9]. However, the simple hypothesis that muscle tension around the cervical vertebra causes tension-type headaches has been criticized [10].

People with tension-type headaches often complain of pain in the head and cervical muscles, but in many cases, they also complain of opening disorders, sprains of the temporomandibular joint, and joint pain $[11,12]$. Temporomandibular joint disorder is a major cause of pain in the oral and facial area, and includes symptoms and habits of oral dysfunction and morphological abnormalities [13]. Previous studies have reported that approximately $70 \%$ of patients with temporomandibular joints complain of headaches [3].

In the previous study, the treatment of tension-type headaches was considered a problem of the cervical vertebra and cervical muscles, and the effect of this study and the relationship between the temporomandibular joint and headache have been investigated but are insufficient. The purpose of the current study was to investigate the effects of temporomandibular joint treatment for tension-type headaches and its effect on pain and functional improvement.

\section{Methods}

\section{Research subjects}

This study included 33 persons diagnosed with tension-type headaches at the Yonsei Balance Clinic in Yangcheon-gu, Seoul from October 21, 2019, to November 9,2019 . The presence of two or more of the following four symptom groups are required to diagnose tension-type headaches according to the International Headache Society classification [9], which is shown in Table 1. Those who had received treatment for the previous 6 months, experienced cervical fractures or surgery, or who were taking injections or drugs were excluded from the study. After the procedure of the experiment was fully explained, the participants provided their voluntary informed consent. This study was conducted with the approval of the Institutional Review Board of Eulji University (IRB No. [on]-19-A-00002950) (Figure $1)$.

\section{Research methods}

\section{Experimental method}

Patients who agreed to participate in the study were randomly selected and assigned to the temporomandibular joint and cervical vertebra treatment group, the temporomandibular joint and general physiotherapy group, or the cervical vertebra and general physiotherapy group, respectively. Eleven subjects were assigned to each group. Treatment was performed for 50 minutes three times a week

Table 1. Diagnostic criteria of chronic tension-type headache (international headache society)

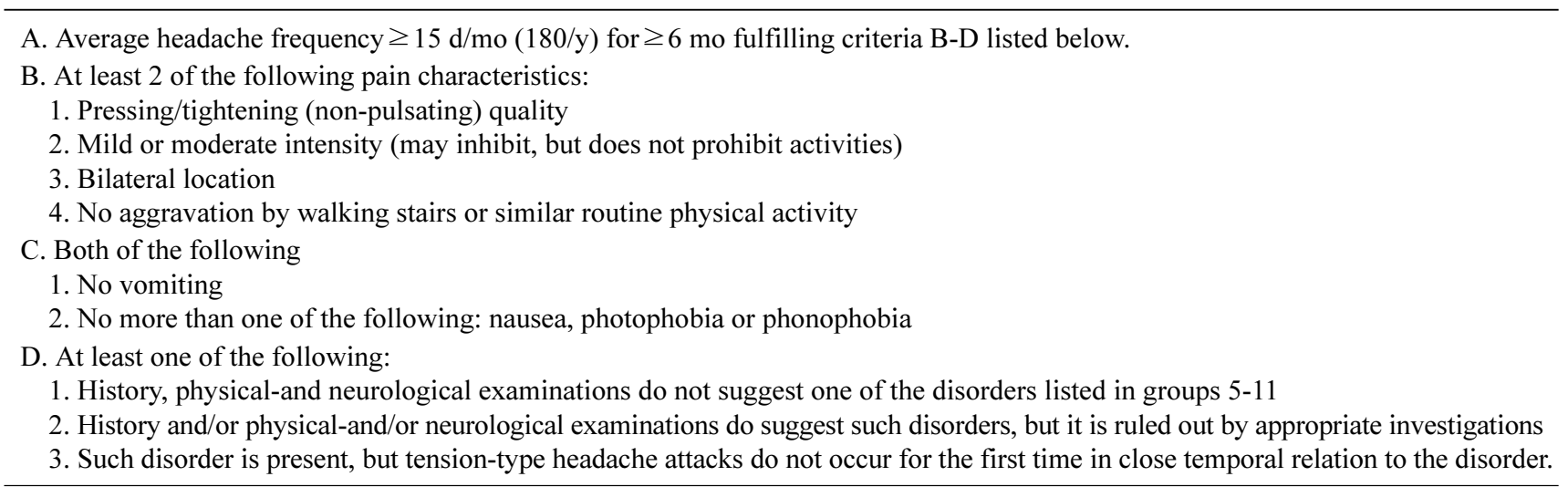




\begin{tabular}{|c|c|c|}
\hline \multicolumn{3}{|c|}{ Subjects ( $n=33$ ) } \\
\hline TCG $(n=11)$ & TG $(n=11)$ & $C G(n=11)$ \\
\hline \multicolumn{3}{|c|}{ Pre-test $(n=33$ ) ( SF-MPQ,K HIT-6 PrLPrR NDI ) } \\
\hline $\begin{array}{c}\text { TCG } \\
\text { (Temporomandibular } \\
\text { joint and cervical } \\
\text { vertebra) }\end{array}$ & $\begin{array}{c}\text { TG } \\
\text { (Temporomandibular } \\
\text { joint+General } \\
\text { Physiotherapy) }\end{array}$ & $\begin{array}{c}\text { CG } \\
\text { (cervical } \\
\text { vertebra } \\
\text { +General } \\
\text { Physiotherapy) }\end{array}$ \\
\hline \multicolumn{3}{|c|}{ Post-test $(n=33)$ ( SF-MPQ,K HIT-6 PrLPrR NDI ) } \\
\hline \multicolumn{3}{|c|}{ Data analysis ( SPSS window version 20) } \\
\hline
\end{tabular}

Figure 1. Diagram of the study. TCG: temporomandibular joint and cervical vertebra group, TG: temporomandibular joint group, CG: cervical vertebra group, SF-MPQ, K: Korean version of the short form-McGill Pain Questionnaire, HIT-6: Headache Impact test-6, PrL: pressure pain left, PrR: pressure pain right, NDI: Neck Disability Index. for four weeks.

\section{Treatment of the temporomandibular joint}

Treatment of the temporomandibular joint was performed for 25 minutes. Treatment consisted of pain points of chewing muscles, relaxation and stretching of shortened muscles, and treatment of joint mobility limitations. The main targets were the frontalis and temporalis, pterygoid, and masseter muscles. Deep tendon massage, myofascial release, and direct omnidirectional mobilization of the temporomandibular joint were performed on the medial and lateral pain areas (Figure 2) [13].

\section{Cervical vertebra treatment}

Cervical vertebra treatment was performed for 25 minutes. The cervical vertebra was treated by performing deep tendon massage on the splenius capitis, trapezius, scalene, levator scapular, and sternocleidomastoid (SCM) muscles, myofascial release on the soft tissue below the larynx, and arthroplasty on the most painful areas of the neck joint (Figure 2) [7].

\section{General physiotherapy}

General physical therapy was performed for 25 minutes. Percutaneous irritation treatment was applied for 15 minutes with a hot pack. Treatment was applied to the splenius capitis, trapezius, SMC, and levator scapular sites.

\section{Measurements}

The Korean version of the short form-McGill Pain

\section{Questionnaire}

Korean version of the short form-McGill Pain Questionnaire (SF-MPQ, K) has been shown to have good reliability in previous studies (intra-class correlation coefficient [ICC] $=$ 0.809). The SF-MPQ, $\mathrm{K}$ is divided into three parts and consists of adjectives representing 15 pain states. This questionnaire can be completed in a short time without the help of medical staff.

\section{Headache Impact test- 6}

The HIT-6 is a questionnaire that examines the effects of headache on daily life. HIT- 6 has been shown to have good reliability in previous studies ( $\mathrm{ICC}=0.76-0.83$ ) [14]. The higher the test score, the more severe the headache, where a score of greater than 60 indicates a severely impaired life. A score below 60 indicates a condition of having little or no obstacles in daily life.

\section{Digital algometer FPX25}

The Digital Algometer FPX25 (Wagner Instruments, Greenwich, CT, USA) is a tool for measuring the threshold of pain. When the soft tissue is pressed with a pressure catheter, the pressure value at the moment of discomfort or pain in the pressed area is measured. To assess the headache area, the participant pointed to the area where he or she felt the headache. The test administrator placed the Digital Algometer FPX25 on this area and recorded the value when the subject felt discomfort or pain. The average value was determined by measuring each region twice. The Digital Algometer 

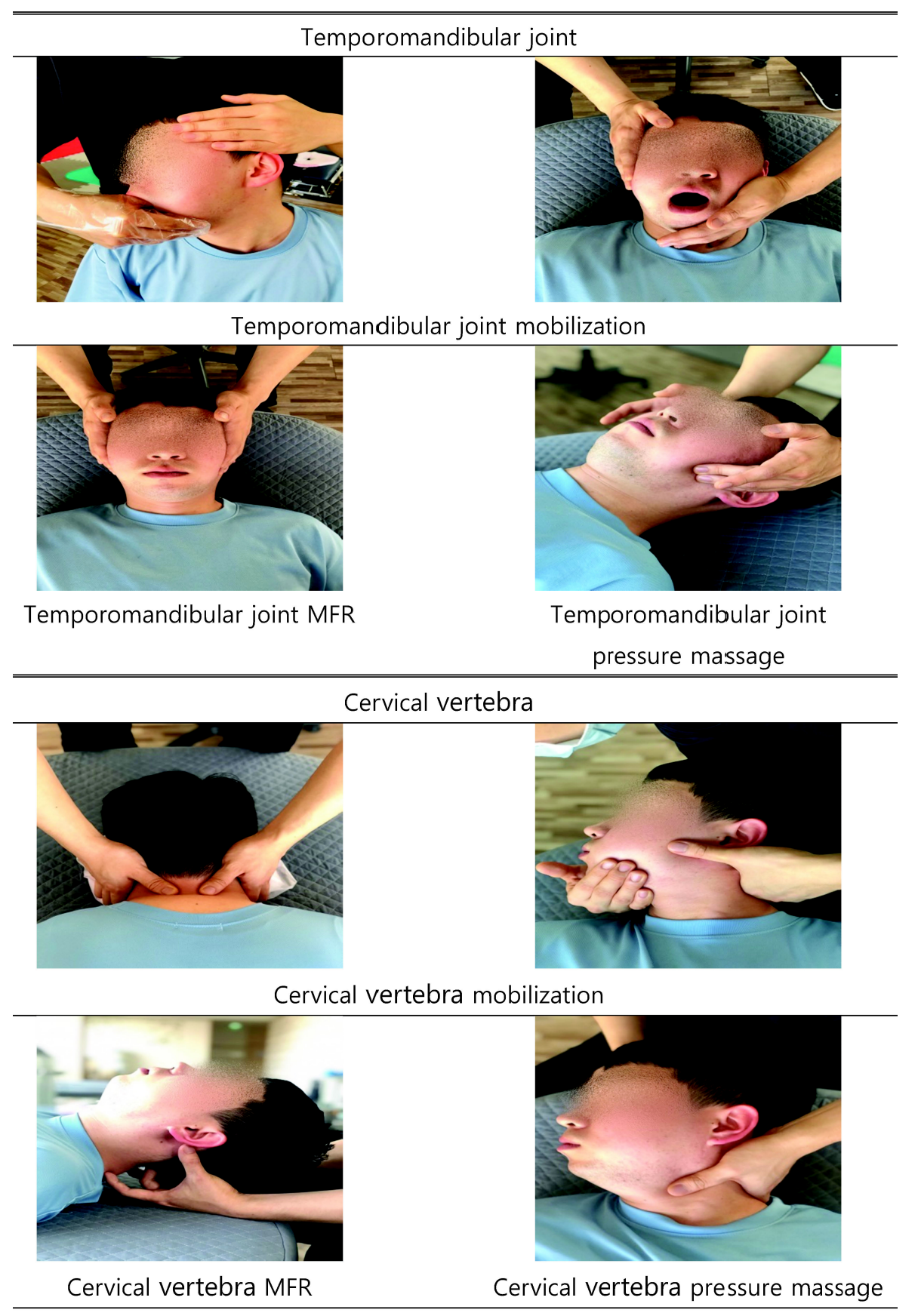

Figure 2. Temporomandibular joint and cervical vertebra treatment. MFR: MyoFascial Release.

FPX25 has been shown to have high reliability $(\mathrm{ICC}=0.78$ 0.93 ) in measuring soft tissue pain [15].

\section{Neck Disability Index}

The Neck Disability Index (NDI) questionnaire has a high reliability (ICC=0.59-0.89) for neck disorder measurements [16]. The questionnaire consists of a total of 10 questions, where later questions are given higher scores. It evaluates pain intensity, daily life activities, reading, lifting, head- aches, concentration, driving, work, sleep, and leisure life. A score of 0-8 indicates no disability, 10-28 indicates a slight disability, 30-48 indicates a moderate disability, 50-68 indicates a severe disability, and more than 70 indicates a complete disability [17].

\section{Data analysis}

The measured data were statistically analyzed using IBM SPSS Statistics for Windows, Version 20.0 (IBM Co., Armonk, 
NY, USA). One-way ANOVA was used to compare the characteristics of the three groups, and the paired t-test was used to compare the groups before and after temporomandibular joint and cervical vertebra treatment. One-way ANOVA was used to test for differences in pain patterns, pain thresholds, headaches, and NDI, and the Bonferroni method was used for a post-hoc test. The statistical significance level was set to $\mathrm{a}=0.05$.

\section{Results}

\section{General characteristics of subjects}

The characteristics of the study subjects were as follows: Thirty-three subjects with tension-type headaches were included. The temporomandibular joint and cervical vertebra group (TCG) consisted of four males and seven females, and the temporomandibular joint group (TG) consisted of three males and eight females. There were no significant differences among the groups in the characteristics of the participants. General characteristics of the study subjects are presented in Table 2.

Comparison of the Korean version of the short formMcGill Pain Questionnaire and Headache Impact test-6 measurement results

A comparison of the SF-MPQ, K and HIT-6 scores before and after intervention among the three groups is presented in Table 3. Significant differences in SF-MPQ, K scores before and after intervention were found in the TCG, TG, and CG $(p<0.05)$. Significant differences in HIT-6 score before and after intervention were found in the TCG, TG, and CG $(p<$ $0.05)$. A comparison between the three groups before and after intervention is presented in Table 3. Significant differences in the HIT-6, SF-MPQ, K scores after intervention were found between the groups $(p<0.05)$. Post-hoc tests revealed that the TCG was significantly different from the TG and CG in all the variables $(p<0.05)$. The SF-MPQ, K, and
HIT-6 scores were not significantly different between the TG and CG $(p>0.05)$.

\section{Comparison of measurement results of the Digital Algo- meter FPX25 and NDI}

A comparison of the Digital Algometer FPX25 scores and NDI before and after intervention among the three groups is presented in Table 3. In the TCG, TG, and CG, significant differences were found in the left and right values of the Digital Algometer FPX25 before and after intervention

Table 3. Comparison before and after intragroup intervention $(\mathrm{N}=33)$

\begin{tabular}{cccr}
\hline Variable & Pre & Post & \multicolumn{1}{c}{$\mathrm{t}(p)$} \\
\hline \multicolumn{2}{c}{ SF-MPQ, K (score) } & & \\
TCG & $35.36(0.92)$ & $22.64(1.80)$ & $19.23(0.001)$ \\
TG & $35.18(1.25)$ & $26.45(2.42)$ & $9.63(0.001)$ \\
CG & $35.36(1.74)$ & $26.18(1.66)$ & $14.57(0.001)$ \\
HIT-6 (score) & & & \\
TCG & $57.91(2.50)$ & $44.09(3.27)$ & $9.52(0.001)$ \\
TG & $59.00(2.56)$ & $51.64(2.69)$ & $12.19(0.001)$ \\
CG & $57.91(2.21)$ & $50.64(2.65)$ & $20.25(0.001)$ \\
PrL (score) & & & \\
TCG & $15.55(1.80)$ & $19.18(2.04)$ & $23.90(0.001)$ \\
TG & $15.91(1.51)$ & $18.18(1.25)$ & $-6.33(0.001)$ \\
CG & $15.64(1.91)$ & $19.09(1.70)$ & $-7.95(0.001)$ \\
PrR (score) & & & \\
TCG & $14.91(1.64)$ & $19.00(1.18)$ & $-10.43(0.001)$ \\
TG & $14.55(1.57)$ & $16.82(1.77)$ & $-9.58(0.001)$ \\
CG & $15.82(1.72)$ & $18.27(1.48)$ & $-7.86(0.001)$ \\
NDI (score) & & & \\
TCG & $47.82(5.26)$ & $28.45(2.64)$ & $15.46(0.001)$ \\
TG & $51.73(5.62)$ & $41.45(4.78)$ & $14.37(0.001)$ \\
CG & $47.45(6.83)$ & $35.36(5.81)$ & $8.90(0.001)$ \\
\hline
\end{tabular}

Values are presented as mean (SD).

SF-MPQ, K: Korea version of the short form-McGill Pain Questionnaire, HIT-6: Headache Impact test-6, PrL: pressure pain left, PrR: pressure pain right, NDI: Neck Disability Index, TCG: temporomandibular joint and cervical vertebra group, TG: temporomandibular joint group, CG: cervical vertebra group.

Table 2. General characteristics of subjects

\begin{tabular}{lcccc}
\hline \multicolumn{1}{c}{ Variable } & TCG $(\mathrm{n}=11)$ & TG $(\mathrm{n}=11)$ & CG $(\mathrm{n}=11)$ & $\mathrm{F}(p)$ \\
\hline Age $(\mathrm{y})$ & $40.73(7.82)$ & $49.27(11.37)$ & $41.82(7.50)$ & $2.890(0.710)$ \\
Height $(\mathrm{cm})$ & $168.55(6.63)$ & $168.91(5.30)$ & $170.36(5.60)$ & $0.290(0.740)$ \\
Weight $(\mathrm{kg})$ & $63.55(7.89)$ & $66.27(10.18)$ & $63.82(10.13)$ & $0.270(0.760)$ \\
Sex (male/female) & $5 / 6$ & $6 / 5$ & $5 / 6$ & $0.111(0.895)$ \\
\hline
\end{tabular}

Values are presented as mean (SD) or number only.

TCG: temporomandibular joint and cervical vertebra group, TG: temporomandibular joint group, CG: cervical vertebra group. 
Table 4. Comparison before and after intervention

$(\mathrm{N}=33)$

\begin{tabular}{lcccc}
\hline \multicolumn{1}{c}{ Variable } & TCG $(\mathrm{n}=11)$ & TG $(\mathrm{n}=11)$ & $\mathrm{CG}(\mathrm{n}=11)$ & $\mathrm{F}(p)$ \\
\hline SF-MPQ, K (score) & $12.72(2.19)^{*}$ & $8.72(3.00)$ & $9.18(2.08)$ & $8.696(<0.001)$ \\
HIT-6 (score) & $18.18(1.45)^{*}$ & $7.36(0.60)$ & $7.27(0.35)$ & $16.232(0.001)$ \\
PrL (score) & $3.63(0.50)^{*}$ & $2.27(1.19)$ & $3.45(1.43)$ & $4.830(0.015)$ \\
PrR (score) & $4.09(1.30)^{*}$ & $2.27(0.78)$ & $2.45(1.03)$ & $9.785(<0.001)$ \\
NDI (score) & $19.36(4.15)^{*}$ & $10.27(2.37)$ & $12.09(4.50)$ & $17.692(0.001)$ \\
\hline
\end{tabular}

Values are presented as mean $(\mathrm{SD})$.

TCG: temporomandibular joint and cervical vertebra group, TG: temporomandibular joint group, CG: cervical vertebra group, SF-MPQ, K: Korea version of the short form-McGill Pain Questionnaire, HIT-6: Headache Impact test-6, PrL: pressure pain left, PrR: pressure pain right, NDI: Neck Disability Index.

*Significant difference in a comparison with the CG.

$(p<0.05)$. NDI also showed significant differences before and after intervention in the groups $(p<0.05)$. The comparison between the groups before and after each intervention is presented in Table 4. Significant differences in the left and right values of the Digital Algometer FPX25 scores and NDI after intervention were found between the three groups ( $p<$ 0.05). Post-hoc testing revealed that the TCG was significantly different from the TG and CG in all the variables $(p<0.05)$. The TG and CG did not show any significant differences between the left and right values and NDI of the Digital Algometer FPX25 ( $p>0.05)$.

\section{Discussion}

Headaches are the main symptoms for those who have temporomandibular joint abnormalities. Many studies have been conducted to investigate the relationship between the temporomandibular joint dyfunction and headaches [18] and have found that patients with temporomandibular joint dysfunction reported having nearly twice as many headaches as those without temporomandibular joint dysfunction [19]. von Piekartz and Lüdtke [20] resolved shortening of pain incidence and limiting range of motion of the chewing muscles and peripheral cranial muscles among patients with signs of temporomandibular joint insufficiency. As a result, headache and neck bone functioning were improved significantly. This is consistent with treatment of the temporomandibular joint having positive effects on headache and cervical vertebra improvement.

Treating the temporomandibular joint with arthroplasty of the pain-induced points of chewing muscles stimulates local ischemia and intrinsic sensations, shortens muscle relaxation, and limits the range of motion of the temporomandibular joint [21]. It is thought that this method can relax the fi- brous adhesions to promote synovial circulation and reduce pain. Problems associated with tension-type headaches include tension in the muscles of the lower back, the cranial periphery, and the cervical vertebra [22]. The muscles around the cranial and cervical vertebrae include the frontalis, masseter, pterygoid, SCM, temporalis, splenius capitis, scalene, and the levator scapular muscles. In a previous study, 145 patients with tension-type headaches reported significant reduction in pain intensity after joint mobilization, manual therapy, and myofacial release [23]. Park and Hwang [24] significantly improved their headache and neck disability indices after a four-week relaxation approach in 35 patients with tension-type headaches. This is consistent with the treatment of the cervical vertebra in this study having positive results on pain and functional improvement. This can help restore the ideal length of the fascia by increasing the fascia complex with manual therapy and myofacial release [25].

It is thought that pressure decreases as the state of shortened tissue and connective tissue improves as the rigid fascia relaxes. The results of this study showed that temporomandibular joint and cervical vertebra treatment were effective in reducing headaches, based on the SFMPQ, K, HIT-6, and NDI scores. It was also found that this treatment was effective in improving neck function, and that the sensitivity of the left and right tenderness threshold was reduced. In a previous study, applying the fascia massage technique resulted in a change in neck tension and pain as the irregular muscles and fascia were relaxed [26]. After 4 weeks, patients reported a decrease in headaches after myofacial release and manual therapy treatment [27]. In addition, a previous study on the treatment of the temporomandibular joint and facial muscles found that treating the temporomandibular joint and facial muscles resulted in a significant differ- 
ence in headache pain, cervical function, and quality of life compared to the control group [20].

In our study, we found that the group treated for the temporomandibular joint and cervical vertebra showed significant differences in the SF-MPQ, K, HIT-6, and NDI scores, compared to the treatment groups of either the temporomandibular joint or cervical vertebra groups alone $(p<0.05)$. The left and right tenderness threshold was also significantly different between the TCG and the CG and TG participants $(p<0.05)$. In people with tension-type headaches, many of them complain of pain in the temporal and submandibular joints, as well as around the cranium, neck, face, and ears, which control relaxation of the tense muscle groups around the neck and temporomandibular joint. By treating the temporomandibular joint and cervical vertebra in combination, it resolves the problems related to mastication that are not resolved by cervical vertebra treatment, as well as problems with lower back tension that is not resolved by temporomandibular joint treatment. Therefore, treating the temporomandibular joint and cervical vertebra in conjunction is more effective than treating either the temporomandibular joint or cervical vertebra alone, in terms of improving pain and function in persons with tension-type headaches.

The limitations of this study were the small sample size and the short study period. In addition, it was not possible to fully control variables other than treatment time, particularly the study participants' drug use. Future studies will require a larger cohort and a longer treatment period.

The purpose of this study was to investigate the effects of temporomandibular joint and cervical vertebra treatment on pain, tenderness, and functional improvement in persons with tension-type headaches. In this study, the combined treatment of the temporomandibular joint and cervical vertebra was shown to be effective for improving pain, quality of life, and cervical vertebra in persons with tension-type headaches. These results may serve to be helpful in identifying appropriate treatment for those with tension-type headaches in the future.

\section{Conflict of Interest}

The authors declared no potential conflicts of interest with respect to the authorship and/or publication of this article.

\section{References}

1. Chatchawan U, Thongbuang S, Yamauchi J. Characteristics and distributions of myofascial trigger points in individuals with chronic tension-type headaches. J Phys Ther Sci 2019;31:306-9.

2. Kang KW, Kang DW, Kwon GY, Kim HB, Noh KM, Baek GH, et al. The impact of head repositioning accuracy and proprioception on cervical stabilization exercise in healthy adults. Phys Ther Rehabil Sci 2015;4:49-54.

3. Headache Classification Committee of the International Headache Society (IHS). The International Classification of Headache Disorders, 3rd edition (beta version). Cephalalgia 2013;33: 629-808.

4. Mathew NT. The prophylactic treatment of chronic daily headache. Headache 2006;46:1552-64.

5. Yim J, Park J, Kim H, Woo J, Joo S, Lee S, et al. Comparison of the effects of muscle stretching exercises and cupping therapy on pain thresholds, cervical range of motion and angle: a cross-over study. Phys Ther Rehabil Sci 2017;6:83-9.

6. Grazzi L, Andrasik F, D'Amico D, Leone M, Usai S, Kass SJ, et al. Behavioral and pharmacologic treatment of transformed migraine with analgesic overuse: outcome at 3 years. Headache 2002;42:483-90.

7. Park K. The effect of manipulation therapy on tension-type headache recovery [Master thesis]. Daegu: Daegu University; 2005.

8. Lee H, Shim J, Oh D. Effects of high-frequency diathermy integrated into suboccipital release on tenderness and neck mobility and disability in people with chronic tension-type headache. Phys Ther Korea 2017;24:37-47.

9. Kim J. The effect of cervical spine manipulation on tension-type headache [Master thesis]. Suwon: Kyonggi University; 2010.

10. Park H, Bea S, Yoo S, Jeon Y, Hong J, Uh K. Comparison of tension type headache associated with pericranial tenderness and headache attributed to temporomandibular joint disorder using RDC/TMD axis II . Korean J Oral Med 2010;35:123-33.

11. Lee H, Hong J, Chun Y. Orofacial evaluation of tension-type headache in dentistry. J Oral Med Pain 2004;29:395-406.

12. Kim JH, Kim JG, Do KS, Yim J. The effect of applying a head-weight device on cervical angle and pain of neck muscles. Phys Ther Rehabil Sci 2016;5:101-5.

13. Woo JM. Treatment of temporomandibular disorders alleviates symptoms of tension-type headaches associated with pericranial tenderness: a randomized controlled study. Seoul: Sahmyook University; 2016

14. Kosinski M, Bayliss MS, Bjorner JB, Ware JE Jr, Garber WH, Batenhorst A, et al. A six-item short-form survey for measuring headache impact: the HIT-6. Qual Life Res 2003;12:963-74.

15. Ylinen J, Nykänen M, Kautiainen H, Häkkinen A. Evaluation of repeatability of pressure algometry on the neck muscles for clinical use. Man Ther 2007;12:192-7.

16. MacDermid JC, Walton DM, Avery S, Blanchard A, Etruw E, McAlpine $\mathrm{C}$, et al. Measurement properties of the neck disability index: a systematic review. J Orthop Sports Phys Ther 2009;39: 400-17.

17. Vernon H, Mior S. The Neck Disability Index: a study of reliability and validity. J Manipulative Physiol Ther 1991;14:40915. 
18. Gonçalves DA, Bigal ME, Jales LC, Camparis CM, Speciali JG. Headache and symptoms of temporomandibular disorder: an epidemiological study. Headache 2010;50:231-41.

19. Ciancaglini R, Radaelli G. The relationship between headache and symptoms of temporomandibular disorder in the general population. J Dent 2001;29:93-8.

20. von Piekartz H, Lüdtke K. Effect of treatment of temporomandibular disorders (TMD) in patients with cervicogenic headache: a single-blind, randomized controlled study. Cranio 2011;29:4356.

21. Travell JG, Simons DG. Myofascial pain and dysfunction: the trigger point manual: the upper extremities. Baltimore: Williams \& Wilkins; 1983.

22. Palomo MS, del Barrio MA, Aroca IL-Z, Pascual FH, Izquierdo AY. Cefalea (I). Cefalea tensional. Headache (I). Tension headaches. Med-Progr de Form Méd Contin Acreditado 2015;11: 4147-54.
23. Castien RF, van der Windt DA, Blankenstein AH, Heymans MW, Dekker J. Clinical variables associated with recovery in patients with chronic tension-type headache after treatment with manual therapy. Pain 2012;153:893-9.

24. Park SY, Hwang S. Effects of relaxation approach with self-exercise on head posture, static postural stability, and headache in persons with tension-type headache. Phys Ther Rehabil Sci 2016;5:178-84.

25. Schleip R. Fascial plasticity - a new neurobiological explanation: part 1. J Bodyw Move Ther 2003;7:11-9.

26. Cheon $\mathrm{S}$. The effects of myofascial meridians therapy on changes in slope of cervical vertebrae [Master thesis]. Seoul: Soongsil University; 2012.

27. Ajimsha MS. Effectiveness of direct vs indirect technique myofascial release in the management of tension-type headache. $\mathrm{J}$ Bodyw Mov Ther 2011;15:431-5. 